\title{
Manual de Política Normativa Para una Institución de Educación a Distancia
}

\author{
Pedro Antonio Vela González Ed. D. \\ Vicky Del Rosario Ahumada De La Rosa Ed. D. ${ }^{2}$ \\ José Humberto Guerrero Rodríguez Ed. D. ${ }^{3}$
}

Recibido: $21-07-2017$

Aceptado: 27-09-2017

\section{RESUMEN}

En las instituciones educativas que ofertan programas bajo cualquier modalidad de educación, se hace necesario el análisis, diseño, desarrollo, implementación y evaluación de un manual de política normativa que permita el aseguramiento de la calidad educativa impartida. En esta revisión literaria se presentan criterios básicos a considerar por parte de una Institución de Educación a Distancia (IED) en lo que respecta a las generalidades, condiciones y componentes de las áreas filosófica, jurídica, fiscal, geográfica y de dirección académica, administrativa y financiera, tecnológica, profesoral y estudiantil, elementos que constituyen el manual de política normativa. Se presentan como anexo,

1. Doctor en Educación NOVA Southeastern University - EUA, Magister en Educación U. Javeriana, Magister en Educación U. Santo Tomás, Especialista en Educación U. Santo Tomás, Especialista en Evaluación en Entornos Virtuales de Aprendizaje U. de Granada España, Licenciado en Química U. Pedagógica Nacional. Investigador del Grupo Ambientes de Enseñanza Aprendizaje de las Ciencias Básicas (AMECI), categorizado por Colciencias y avalado por la Universidad Nacional Abierta y a Distancia (UNAD) y la Universidad Distrital Francisco José de Caldas (UDFJC).

Correo electrónico: pedro.vela@unad.edu.co

ORCID: 0000-0001-6990-9026

2. Doctor en Educación NOVA Southeastern University - EUA, Magister en Administración y Supervisión Educativa U. Externado de Colombia, Especialista en Pedagogía para el Desarrollo del Aprendizaje Autónomo U. Nacional Abierta y a Distancia (UNAD) y Licenciada en Ciencias de la Educación U. del Atlántico. Docente de la Escuela Ciencias de la Educación en la UNAD. Investigadora del Grupo Ambientes de Enseñanza Aprendizaje de las Ciencias Básicas (AMECI), categorizado por Colciencias y avalado por la Universidad Nacional Abierta y a Distancia (UNAD) y la Universidad Distrital Francisco José de Caldas (UDFJC).

Correo electrónico: vicky.ahumada@unad.edu.co

ORCID: 0000-0002-8797-331X

3. Doctor en Educación Nova Southeastern University - EUA, Maestría en Docencia Universitaria U. de la Salle y Químico U. Nacional de Colombia. Investigador del Grupo Ambientes de Enseñanza Aprendizaje de las Ciencias Básicas (AMECI), categorizado por Colciencias y avalado por la Universidad Nacional Abierta y a Distancia (UNAD) y la Universidad Distrital Francisco José de Caldas (UDFJC).

Correo electrónico: jose.guerrero@unad.edu.co

ORCD: 0000-0002-8252-6473 
algunos referentes en relación con la política normativa para Colombia que, con su obligatoriedad en la aplicación, permite garantizar por parte de las instituciones educativas la prestación de un servicio de alta calidad.

Palabras clave: Políticas normativas, educación a distancia, calidad educativa, diseño instruccional, normatividad institucional.

\title{
Normative Manual for a Distance Learning Institution
}

\begin{abstract}
In educational institutions that offer programs under any form of education, it is necessary to analyze, design, develop, implement and evaluate a normative policy manual that allows for the assurance of the educational quality imparted. This literary review presents basic criteria to be considered by an Institution of Distance Education (IDE) in terms of the generalities, conditions and components of the philosophical, legal, fiscal, geographical and academic, administrative and Financial, technological, professorial and student that constitute the normative policy manual. Some references are presented as an annex in relation to the normative policy for Colombia, which, with its mandatory application, makes it possible to guarantee the provision of a high quality service by educational institutions.
\end{abstract}

Keywords: Normative policies, distance education, educational quality, instructional design, institutional regulations.

\section{Introducción}

La Educación a Distancia es una forma de impartir educación institucionalizada para brindar a muchas personas oportunidades para desarrollar procesos formativos conjuntamente con compromisos 
sociales y laborales que ayudan a viabilizar una sociedad y su cultura (Miklos, \& Arroyo, 2008). Este documento desarrolla la propuesta de un manual de normas para una Institución de Educación a Distancia, IED.

Tiene en cuenta la normatividad nacional, para el caso de Colombia, en materia de educación (Ley 30 de 1992, Ley 115 de 1994, Ley 1188 de 2008, decreto 1075 de 2015), recopilada por Rubio y González (2011), cuyos textos se encuentran en los apéndices de este documento ya que la IED ofrece programas en educación básica, media y superior.

Las políticas se refieren al marco de acción que definen roles y responsables en la operación del sistema de Educación a Distancia (Simonson, 2007) y ellas deben responder a las políticas establecidas por el Estado por ser la educación una de sus áreas estratégicas que si bien la vigila, le da condiciones de autonomía para que cada uno de los proyectos educativos que se propongan ayuden a su desarrollo en beneficio del bien común (Fliguer, 2010).

Para la IED es la formulación de su compromiso de alinearse con los propósitos de la Educación a Distancia como son facilitar el aprendizaje individualizado, el uso de una amplia variedad de medios tecnológicos para entregar y hacer la instrucción, no establecer distinciones, desigualdades o privilegios de ninguna clase (sexo, religión, credo político, nivel socioeconómico, etnia y otros que limiten el acceso a los programas), facilitar la comunicación sin importar tiempo y lugar, fomentar el autoaprendizaje, tener una organización sistémica y unos costos razonables (Lampert, 2000). En esta forma es posible para el estado y la sociedad tener mecanismos de inclusión social a través de la educación que puede realizarse con Educación a Distancia por facilitar mayor cobertura, flexibilidad en los medios y estrategias instruccionales por utilizar y su capacidad de atender poblaciones heterogéneas (Matias, 2008).

\section{Objetivo del Manual de Políticas Normativas}

El manual de políticas normativas para una IED, tiene como objetivo brindar una guía a las autoridades, a los integrantes de la comunidad educativa y a otros, en la toma de decisiones respecto a las condiciones que definen la operacionalización del sistema de Educación a Distancia, 
asegurando su alineación con la misión, visión y las políticas de calidad de la organización educativa y con ello salvaguardar la calidad de los procesos académicos ofrecidos, respondiendo a las políticas educativas establecidas por el estado en función de la calidad educativa, definida ésta como el ofrecimiento de la mejor educación posible a los estudiantes para contribuir, en última instancia, al bien de la sociedad (Valenzuela, 2010).

\section{Políticas Normativas de la Institución de Educación a Distancia}

Para King, Nugent, Eich, Mlinek y Russell (2000) una política es un curso de acción escrito, tal como un estatuto, procedimiento, regla o regulación, que se adopta para facilitar el desarrollo de un programa académico. Simonson, Smaldino, Albright \& Zvacek, (2012) plantean que en toda IED antes de comenzar a ofrecer cursos a distancia o en línea desarrolle su manual de políticas normativas que facilitará el proceso de desarrollo e implementación de estos programas. Watson y Gemin (2009) recomiendan definir e identificar a quién va dirigidas las políticas de un manual cuando se refiere a procesos académicos en una IED.

Para el desarrollo de la estructura del manual de políticas para la educación a distancia, se sigue los modelos propuestos por King et al., (2000) y Gellman-Danley y Fetzner (1998) que consideran siete áreas estratégicas: (a) filosófica; (b) jurídica; (c) fiscal, geográfica, y de dirección; (d) académica; (e) tecnológica; (f) profesoral; y (g) para los estudiantes.

\section{Política Filosófica}

Para la IED es de relevancia que en este componente se tengan en cuenta la misión, visión, creencias y valores institucionales. Simonson (como se citó en Moore, 2007), afirma que cuando una organización educativa en la modalidad a distancia decide ofertar programas, logra su éxito cuando el reconocimiento que la sociedad hace de la misión y visión sean de alta calidad, creíbles y adecuadas.

Se presentan entonces los componentes de las condiciones para el establecimiento de las políticas filosóficas para la IED. 
Misión de la IED. Proporciona a las personas conocimientos y oportunidades educativas a través de la modalidad abierta y a distancia, que le permitan satisfacer sus necesidades de aprendizaje facilitándoles el acceso al conocimiento, más allá y por encima de la planificación habitual y las restricciones geográficas en el marco de la sociedad global y del conocimiento propiciando el desarrollo económico, social y humano sostenible de las comunidades locales, regionales y globales con calidad, eficiencia y equidad social.

Visión de la IED. La IED incorpora a personas que ofrecen una educación universitaria bajo la modalidad a distancia y de alta de calidad promoviendo: (a) una educación innovadora que permite el aprendizaje personalizado, (b) un liderazgo tecnológico y el trabajo en colaboración, (c) una investigación académica acerca de la sociedad de la información y el aprendizaje electrónico (e-learning) y (d) la diseminación del conocimiento.

Creencias. Las creencias son estructuras de pensamiento elaboradas y arraigadas a lo largo del aprendizaje que sirven para explicar la realidad y que preceden a la configuración de valores. Las creencias responden a las preguntas: ¿En qué creemos? ¿Qué principios nos rigen? Son además principios que las personas y las instituciones van construyendo y sobre las cuales se cimentan sus valores. Al desarrollar las creencias se determina que es lo relevante. Es así, como al expresar la importancia de tener en cuenta diversos puntos de vista y construir sobre ellos, se está dando valor al trabajo en equipo o al expresar el convencimiento de que un ambiente de confianza y respeto debe primar en la IED y aclarando que es un valor institucional que ayuda a mantener un clima positivo.

Decálogo de Valores. En la IED se promueven algunos de los siguientes valores:

1. La calidad, como cultura organizacional.

2. El respeto como elemento básico para su autorrealización personal y profesional.

3. La diversidad de género, la promoción y la aceptación del pluralismo cultural, el multilingüismo para superar barreras y el trabajo participativo mediante distintas plataformas tecnológicas.

4. La innovación, como principio transversal de sus actividades. 5. La sostenibilidad, como base para el diseño y la construcción 
de las actividades y los procesos de la universidad para seguir siendo competitivos.

6. La cooperación, como cultura organizativa basada en la flexibilidad y el compromiso social forjando enlaces institucionales de colaboración, aprendizaje mutuo y trabajo en equipo.

7. La libertad de acción, de pensamiento, de culto y de ideas políticas, pilares para una convivencia pacífica, solidaria y tolerante.

8. La necesidad de cualificar a los estudiantes, docentes, consejeros y funcionarios de todo nivel logrando una sociedad equitativa, justa y emprendedora.

9. El potencial creativo, en la actitud crítica, en el trabajo arduo y honesto de toda la comunidad universitaria.

10. La vivencia de los valores, la ética, el respeto, la disciplina, el debate, la concertación y la conciliación dentro de la comunidad universitaria y otros actores sociales del país.

Principios. Son un marco de referencia permanente en la IED para mejorar la calidad educativa. Algunos de estos principios son:

1. El proceso de enseñanza-aprendizaje centrado en el estudiante.

2. Los cursos académicos están diseñados en Ambientes Virtuales de Aprendizaje (AVA)

3. El fomento de aprendizajes significativos.

4. La libre expresión oral y escrita de los estudiantes a través de los recursos tecnológicos dispuestos en el curso académico. 5. La práctica de los valores dentro de la comunidad académica, entre ellos el respeto por el otro.

6. Tener en consideración los conocimientos previos de los estudiantes.

7. Desarrollar las competencias, habilidades y destrezas con los contenidos adecuándolos a la realidad de los estudiantes y del país.

8. Respetar la multiculturalidad y promover la interculturalidad. 9. Fomentar la evaluación formativa.

10. Considerar los perfiles socioculturales y académicos de los estudiantes y docentes.

11. El aprendizaje debe ser colaborativo. Para Johnsony Johnson (1998) el aprendizaje colaborativo se centra en el proceso 
de construcción del conocimiento a través del aprendizaje que resulta de la interacción con un grupo y mediante tareas realizadas en cooperación con otros. Así mismo es ante todo, un sistema de interacciones cuidadosamente diseñado que organiza e induce la influencia recíproca entre los integrantes de un equipo.

\section{Política jurídica}

La IED por su naturaleza y el uso de las tecnologías de información y comunicación que se incorporan de manera directa con las actividades de creación y producción de su talento humano relacionadas con la actividad académica, investigativa y de producción de bienes y servicios, se ajusta al ordenamiento jurídico nacional e internacional que rige sobre la propiedad intelectual. Las ramificaciones legales en las IED deben incluir aspectos como derechos de autor, uso justo, la responsabilidad por los mensajes electrónicos inapropiados y otras complejidades (GellmanDanley y Fetzner, 1998).

Propósito. La IED orientará el uso de los productos objeto de reconocimiento de propiedad intelectual en coherencia con su misión institucional. Será legítimo el uso de estos productos en el logro de una educación para todos con calidad a través de sus programas de formación investigación, extensión y proyección social.

Definición. Se entiende por propiedad intelectual el conjunto de derechos y prerrogativas sobre todas las creaciones del ingenio humano que, en cualquier campo del saber, puedan ser objeto de creación, definición, reproducción, utilización o expresión por cualquier medio de divulgación.

Contenido. Se establecen las siguientes políticas:

1. La IED fomentará la cultura de la propiedad intelectual, mediante el abordaje y análisis de diferentes aspectos y enfoques relacionados con el tema.

2. La IED integrará un comité encargado de realizar los trámites necesarios para favorecer el registro y la solicitud de licencias de uso de obras que involucren propiedad intelectual.

3. La IED realizará convenios con entes legales para recibir 
asesoría sobre los derechos y obligaciones del uso de la autoría intelectual.

4. La IED realizará sus propios estatutos y procesos sobre el derecho de autoría intelectual, con el fin de facilitar los trámites dentro y fuera de la institución.

5. Los lineamientos de autoría intelectual estarán orientados hacia normativas internacionales, a fin de diversificar su acceso y elevar el nivel académico en la institución.

\section{Políticas fiscales, geográficas y de dirección}

Según Simonson (2007), estas políticas definen los costos por matrícula y otros servicios académicos, las equivalencias en tiempos completos, la observancia de las normas estatales sobre la institución, las áreas de servicios, las relaciones interinstitucionales, las regulaciones de los consorcios, la contratación, el control administrativo y fiscal, los costos de la administración y el manejo financiero.

Cobertura geográfica. La IED oferta sus programas para un territorio nacional específico, pero permite la matrícula de estudiantes de otras nacionalidades observando la legislación de cada país y los acuerdos internacionales vigentes.

Este mismo criterio se aplica cuando se establezcan convenios de cooperación para el desarrollo conjunto o en consorcio de proyectos con otros países o se hagan negociaciones para el intercambio de programas ofertados permitiendo la internacionalización de la educación. En todos los casos, la IED es la responsable por la administración de esos programas en el País de origen conforme a la legislación vigente.

Dirección académica. El desarrollo institucional de la IED se encuentra en sus unidades académicas quienes proponen, elaboran los diseños curriculares y hacen su acreditación ante los Consejos Académico y Superior de la institución, siendo los responsables de radicarlos ante el Ministerio de Educación Nacional según las características específicas de cada programa dando cumplimiento a la normatividad correspondiente.

Dirección administrativa. Las unidades académicas contarán con un director y un equipo de trabajo de coordinación acorde a las áreas de 
conocimiento que administre y tendrá la posibilidad de vincular expertos temporales para el desarrollo de proyectos específicos, pero habrá un responsable en la unidad que se encargue del desarrollo del proyecto hasta su implementación final. Esto como garantía de aprendizaje para la organización en pro de su desarrollo institucional.

Asuntos financieros. La unidad financiera institucional será el proyecto que una vez aceptado, funcionará como un programa aprobado cuando se reciba el correspondiente registro calificado o resolución de aprobación, dependiendo del tipo de proyecto de formación autorizado.

Cadaunidadacadémicatendrásupresupuestoanualyserála responsable de su ejecución. La gerencia administrativa y financiera y su grupo de planeación apoyarán el desarrollo de los proyectos específicos que cada unidad académica proyecte anualmente estableciendo conjuntamente los cronogramas de ejecución presupuestal del año siguiente, garantizando el manejo transparente y eficiente de los recursos financieros institucionales.

Cada año, se programará el costo fijo por gastos administrativos para cada unidad teniendo como base el director, un asistente administrativo, los coordinadores que estén atendiendo el desarrollo de programas aprobados y en funcionamiento, lo mismo que los proyectos aprobados por el Consejo Académico.

Costos académicos. Corresponde a los ingresos que la IED percibirá por la venta de sus servicios en los diferentes programas de formación aprobados y en funcionamiento. La gerencia administrativa establecerá los costos de matrícula, expedición de certificados de matrícula, cursos matriculados, calificaciones, derechos de grado y demás que los estudiantes requieran para acreditar su condición ante otras entidades.

La matrícula en cada uno de los programas se hará por cursos que tome el estudiante dándole derecho al uso del campus, la tutoría, servicios de consejería y de apoyo a la formación, la biblioteca, los servicios de laboratorio para prácticas, las prácticas profesionales requeridas para el cumplimiento de los requisitos académicos y al desarrollo de su trabajo de grado donde se requiera. Los recursos bibliotecarios que la IED pone al servicio de los estudiantes son fundamentales para que éstos puedan cumplir con sus responsabilidades académicas adquiridas y deben tener acceso a las distintas bibliotecas virtuales y otros recursos disponibles en la Internet ofrecidos por la institución (Bonilla, 2004). 
La IED otorgará como apoyo a sus estudiantes más destacados, becas, pasantías institucionales y monitorias virtuales para apoyar el desarrollo formativo del talento humano en formación y esos estímulos serán reconocidos en su hoja académica y en las certificaciones que la institución expida.

Apoyo financiero. La IED establece alianzas estratégicas con entidades financieras para aquellos estudiantes que soliciten financiación a sus matrículas, la adquisición de tecnología o de materiales requeridos para el desarrollo de sus cursos. Sin embargo, la IED servirá como intermediario en la negociación de los créditos pero no se constituye en parte, quedando en responsabilidad del estudiante la cancelación de los mismos y las obligaciones que las normas de cada País, establezcan para su cobro.

Apoyo tecnológico y de servicios. La IED se compromete a mantener en servicio $365 \times 7 \times 24$ del campus, dispondrá de una mesa de apoyo técnico que resolverá las dificultades que por acceso tengan los estudiantes al campus, pero la disponibilidad de tecnología de conectividad y de computación es responsabilidad exclusiva del estudiante. En aquellas regiones del país que tengan dificultades de acceso, la IED dispondrá de alternativas tecnológicas para remitirles a los estudiantes los recursos necesarios para adelantar su estudio y buscará alianzas para facilitar su conexión, en caso de no ser posible, se establecerán estrategias específicas para que no existan barreras ni discriminaciones para la realización de su labor académica.

Rendición pública de cuentas. La IED establecerá mecanismos de comunicación a través de la red para informar a sus usuarios, organismos de control del Estado y a toda la comunidad los resultados y las innovaciones que haga para mantener un servicio académico y administrativo de excelencia para que se constituya en una estrategia de desarrollo de todas las regiones de la geografía nacional y de otros países.

\section{Políticas académicas}

El área académica provee una guía para el calendario de instrucciones, técnicas de diseño instruccional, criterios y métodos de evaluación, alineación del plan estratégico y medir el éxito de la IED (Restauri, 2004; Piskurich, 2006; Yang, 2006). Esta política corresponde al compromiso con 
el conocimiento, con el sujeto que aprende, los espacios de interacción y las relaciones de poder que generan, la presencia social de los actores en el aprendizaje Garrison, D., \& Anderson, T., 2005), las regulaciones en las interacciones y en la construcción ética del saber, el rol de la tecnología en el proceso pedagógico (Copertari, Sgrecia, \& Segura, 2011). Contiene el marco de acción para el calendario académico, acreditación del programa, calidad de los cursos, evaluación de los cursos y programas, graduación, criterios de admisión y la aprobación y revisión curricular (Simonson, 2007).

El conocimiento. La IED oferta programas en las áreas del conocimiento que son enseñables por medio de Educación a Distancia apoyada en Tecnologías de la Información y la Comunicación (TIC) y contribuye a su desarrollo, a la consolidación de nuevos saberes mediante la investigación y propende por su divulgación a todas las personas, organizaciones y naciones que se quieran unir al proyecto.

El saber se fundamenta en las epistemologías de las disciplinas avaladas por las comunidades científicas y promueve la participación de sus profesores, estudiantes y administrativos en el desarrollo de investigaciones que respondan a las necesidades de los contextos donde se encuentren y produzcan soluciones viables.

El aprendizaje. Para (Ormord, 2012) es entendido como proceso en el cual el sujeto construye activamente nuevas ideas o conceptos basados en conocimientos presentes y pasados. Se forma construyendo nuestros propios conocimientos desde nuestras propias experiencias. El aprendizaje permite al ser humano adquirir los conocimientos, habilidades y destrezas necesarios para poder adaptarse a la realidad de su vida y también transformarla (Klimenko y Alvares, 2009).

Las políticas académicas definidas por la IED, "brindarán todas las condiciones para que se desarrollen competencias metacognitivas que fomenten el aprendizaje autónomo cuyo sistema de autorregulación funciona de modo que le permite satisfacer exitosamente tanto las demandas internas como externas que se le plantean" (Bornas,1994: p.13 ); el aprendizaje significativo entendido como la incorporación de la nueva información a la estructura cognitiva del individuo (Ausubel, 2002) y el aprendizaje colaborativo en el que los estudiantes son quienes diseñan su estructura de interacciones y mantienen el control sobre las diferentes decisiones que repercuten en su aprendizaje (Panitz, 2001), conformando 
comunidades de aprendizaje capaces de identificar problemas y elaborar los procedimientos más viables para su solución. Su énfasis está, "no solo en la generación de conocimiento, sino en utilización del conocimiento generado para transformar las estructuras sociales que conlleven a la superación del ser humano" (Ortiz, 2010. p.1).

Así mismo, la conformación de redes de aprendizaje con las que se permite un intercambio crítico de conocimientos y experiencias (Reynaga \& Farfán, 2004), son la estrategia fundamental para promover la presencia social de la IED en su campus y en la sociedad del conocimiento. Los profesores y estudiantes serán los líderes que las promuevan para facilitar los intercambios que les ayuden a afianzar sus conocimientos, su participación y su competitividad para solucionar los problemas del entorno donde se encuentren.

El aprendiz. Es el centro del aprendizaje; la IED brindará las estrategias, recursos y desarrollo de habilidades para que sea el responsable motivado y autónomo en la construcción de su saber individual y grupal.

Aspectos como lo que aprenden verdaderamente los estudiantes, el impulso del aprendizaje en relación con la calidad de los docentes, la mejora en los resultados cuando tienen excelentes docentes, han llevado a que los encargados de formular políticas busquen la manera de identificar a los profesores eficaces para que ello permita el aseguramiento de los aprendizajes. "Esta es la condición imprescindible para que los estudiantes y los países reciban los beneficios económicos y sociales de la educación" (Bruns, B. \& Luque, J., 2015, p. 6).

El profesor. Es el responsable del diseño instruccional de los cursos, entendido éste como un modelo de enfoque de sistemas para el análisis, diseño, desarrollo implementación y evaluación de la enseñanza que puede ser utilizado para mejorar el rendimiento estudiantil (Dick, Carey, L., \& Carey, J., 2009); la valoración de los recursos o medios que faciliten el aprendizaje, "la generación de las interactividades de estudiantematerial, estudiante-estudiante, estudiante-profesor, estudianteinstitución que favorecen la presencia social del estudiante en el campus y en la comunidad de aprendizaje del curso, definida ésta" (Palloff \& Pratt, 2007 p. 230) como la interacción entre estudiantes y docentes promoviendo la honestidad, sensibilidad, relevancia, respeto, apertura y apoderamiento, el diseño del sistema de evaluación del aprendizaje del curso, la valoración de los resultados del logro académico del estudiante 
y del mejoramiento de contenidos, estrategias e interactividades del mismo para que cada vez le de mayor autonomía en el aprendizaje a su estudiante.

En los cursos iniciales de los programas a distancia, debe diseñar estrategias comunicacionales para que el estudiante pueda comprender y mejorar sus habilidades para el estudio individual, en grupo y dentro de su comunidad de aprendizaje (Gamboa, Sandoval \& Ahumada, 2017).

La IED tendrá un programa de perfeccionamiento docente que propondrá estrategias para la capacitación, la formación permanente y especializada para que cada vez sean más eficaces en la enseñanza y la guía hacia el aprendizaje autónomo. Flórez (1994), plantea que el concepto de formación docente debe ir más allá del entrenamiento técnico, de una cultura transmisiva y reproductiva, de una práctica docente restringida hasta concepciones más evolucionadas y pertinentes.

En el foro desarrollado para América Latina, Bruns, B. \& Luque, J. (2015), presentan en el documento Excelentes profesores: cómo mejorar el aprendizaje en América Latina y el Caribe, que,

...para formar cuerpos docentes de alta calidad, deben seguirse tres pasos fundamentales -reclutar, desarrollar y motivar mejores profesores-, y se dispone de una importante cantidad de experiencias de reforma en estos tres aspectos dentro y fuera de la región que pueden orientar el diseño de mejores políticas (Bruns, B. \& Luque, J., 2015, p. 2).

Modelo pedagógico. Un modelo pedagógico plantea de forma integral un determinado fenómeno; desde el punto de vista teórico-práctico ofrece "un marco de referencia para entender implicaciones, alcances, limitaciones y debilidades paradigmáticas que se dan para explicarlo" (Gimeno \& Pérez, 1992. p. 448). Con el modelo pedagógico definido por la IED se establecen las condiciones en que se debe diseñar la enseñanza y orientar el aprendizaje y establece las interacciones del subsistema académico de la IED. Su epistemología se fundamenta en el aprendizaje durante toda la vida y en los principios de la Educación a Distancia; el diseño curricular, las estrategias pedagógicas, el desarrollo de los medios pedagógicos y la evaluación del aprendizaje considera los principios del constructivismo social centrado en la actividad del aprendiz. 
El currículo. Para Arredondo (1981) citado por Nagles y Calderón (2016),

El currículo es el resultado de (a) análisis y reflexión sobre las características del contexto, del educando y de los recursos; (b) La definición, tanto explicita como implícita de los fines y los objetivos educativos; (c) La especificación de los medios y los procedimientos propuestos para asignar racionalmente los recursos humanos, materiales, informativos, financieros, temporales y organizativos, de manera que se logren los fines propuestos (Nagles \& Calderón, 2016, p. 61).

Corresponde al proyecto de formación que una vez aprobado mediante su registro calificado se transforma en programa, está en estrecha relación con la epistemología institucional, la Educación a Distancia y los fundamentos del constructivismo. Contiene las metas, las competencias por desarrollar, el plan de curso, los syllabus, los recursos necesarios para su desarrollo y las condiciones requeridas para la obtención del título correspondiente (Arévalo, M., \& Gamboa, A., 2015).

Este proyecto se encuentra documentado y será conocido por los aspirantes, estudiantes, docentes y personal administrativo que lo regenta como base de su trabajo y sobre el cual se rendirá cuentas de los avances, logros alcanzados y planes de mejoramiento continuo para el aseguramiento permanente de su calidad.

El curso. Es la unidad curricular de cualquier programa ofertado por la IED. Se encuentra alineado a los propósitos curriculares ya que efectúa los aportes al desarrollo de competencias profesionales requeridas para el desempeño profesional avalado por el título que se desea obtener.

La IED tendrá asignado a cada curso un profesor titular quien será el responsable de su diseño, actividades básicas, modelo evaluativo, selección de recursos mediados o de su elaboración y del análisis de los logros obtenidos por los estudiantes. Será el líder de la red académica del curso. También dispondrá de profesores auxiliares quienes serán los encargados del trabajo académico de los estudiantes, de su asesoría, las actividades de interacción individual y grupal y de la construcción de las habilidades y destrezas requeridas para el desarrollo de las comunidades virtuales de aprendizaje y de la calificación de las 
actividades programadas en el plan del curso. Al inicio de la asesoría con cada grupo diseñará el syllabus requerido y establecerá medios de comunicación eficaces con sus estudiantes.

Se documenta a través del syllabus o plan de curso que contiene: Propósito, metas de aprendizaje, competencias por desarrollar, calendario de actividades, modelo de evaluación, productos por presentar para demostrar logros, recomendaciones de recursos mediados por utilizar ya sea de repositorios institucionales o de fácil ubicación en bases de datos o reservorios de recursos recomendados por el profesor.

La Evaluación del aprendizaje. Se constituye en un sistema que tiene como propósito obtener información para la toma de decisiones en la promoción de los estudiantes, la excelencia académica de los profesores y el mejoramiento de sus programas ofertados y poder determinar criterios para informar a la sociedad, a sus usuarios y al área de la Educación a Distancia.

Evaluar los aprendizajes alcanzados por los estudiantes permite establecer si los logros son iguales a las metas propuestas, los contenidos estudiados, las estrategias pedagógicas y de aprendizaje fueron adecuadas y si los instrumentos de evaluación utilizados permitieron comprobar las competencias durante la ejecución de las tareas diseñadas para ponerlas en evidencia (Nitko, \& Brookhart, 2011; Bonvecchio, \& Maggioni, 2004; Lamas, 2008; Ferreyra, Castagno, Salgueiro \& Tucci, 2012).

En la evaluación del aprendizaje, durante el desarrollo del curso, tendrá momentos formativos al aportarle información al estudiante de su desempeño para que reflexione sobre sus logros y se esfuerce en mejorar los aspectos débiles en la consolidación de sus conocimientos, estrategias de aprendizaje y de participación en la construcción de su comunidad virtual de aprendizaje.

Una vez finalizado el proceso, los responsables de la evaluación del aprendizaje deben evaluar los resultados: promedio ponderado de estudiantes, porcentajes de aprobación, en global y para cada uno de los programas, además de analizar los resultados de satisfacción de los estudiantes. A partir de la evaluación de estos resultados se deben establecer acciones de mejora. 
La acreditación curricular, de cursos y de la evaluación. Es el mecanismo de certificación de la calidad de los servicios académicos ofertados por la IED. Cada Unidad Académica establecerá los criterios, indicadores y criterios específicos para la certificación interna de sus currículos, planes de cursos, materiales, servicios de apoyo académico al estudiante y uso eficiente de sus recursos constituyéndose en el momento de autoevaluación. Cumplirá los requisitos para la certificación mínima de calidad requerida para obtener los registros calificados o resoluciones de aprobación de sus programas.

La evaluación institucional. Se utilizará para valorar los logros de los programas de formación conforme a la ontología institucional, con el fin de hacer los mejoramientos permanentes requeridos para mantener la calidad de los mismos, lograr la plena satisfacción de los usuarios y apoyar el desarrollo de las regiones donde viven los egresados.

La necesidad de la evaluación institucional se fundamenta en la demanda cada vez más creciente de la sociedad actual por la oferta de servicios de parte de la universidad en función de estándares de calidad y excelencia. Todo lo cual implica favorecer en la universidad aquello que le permita alcanzar sus objetivos de enseñanza, investigación y proyección social (Rodríguez y Zevallos, 2003). Para los mismos autores, evaluar la universidad conlleva emitir un juicio de valor a propósito de la pertinencia y la razón de ser de sus objetivos, su grado de cumplimiento y la forma en que han sido alcanzados. Evidentemente, todo ello constituye un proceso complejo de implementación y desarrollo; caracterizándose por su gradualidad, consensualidad, colegialidad, honestidad y responsabilidad.

Es por ello que la evaluación institucional corresponde a un conjunto de prácticas, métodos, conocimientos bien diversificados y progresivamente desarrollados en el transcurso de los años con el objeto de dar respuesta a una necesidad específica: la de una mejor gestión de la universidad (Vela, Ahumada \& Guerrero, 2016). Ello implica favorecer en la universidad todo aquello que le permita alcanzar sus objetivos de enseñanza e investigación y fortalecer la calidad de sus actividades (Simoneau, 2003).

Para Álvarez (1992) la evaluación institucional en las organizaciones universitarias debe apuntar al mejoramiento de la calidad en el marco del planeamiento estratégico de forma permanente, sistemática, cuyo alcance tiene que ver con los procesos, los productos y su impacto 
en la sociedad. Afirma además que debe ser una tarea constructiva tendiente a ayudar a detectar el déficit y las dificultades en materia académica, recursos físicos, docentes y técnicos, para mejorar la gestión institucional en el logro de las metas, y con ello neutralizarlas y superarlas. Comprendida así la evaluación institucional se convierte en una herramienta importante de transformación de las universidades y de la práctica educativa, en la medida que el propio proceso de la investigación, evaluación y planificación permita explicar y entender los problemas, los objetivos y propósitos de la institución.

Acreditación Institucional de Alta Calidad. La IED fundamenta su éxito en la calidad, por lo que desarrollará estrategias para la acreditación y certificación nacional e internacional de sus programas mediante agencias reconocidas. En Colombia, la normatividad específica establece la acreditación voluntaria ante el Consejo Nacional de Acreditación (CNA).

\section{Política tecnológica}

Para Sandia y Montilva (s.f.), el área Tecnológica proporciona, opera y mantiene toda la infraestructura de equipos y sistemas necesarios para garantizar la comunicación interactiva entre otras áreas. Así mismo, un modelo de soporte debe proporcionar a la IED un entorno tecnológico de vanguardia, con servicios innovadores, de calidad y estables, contribuyendo y añadiendo valor a las actividades educativas de investigación y de gestión de la IED.

Para ello debe considerarse varios equipos de trabajo:

1. Un equipo que se encargue de gestionar los servicios para el aprendizaje. Sus funciones serán: (a) Acompañar al profesorado en la definición, mejora, búsqueda, integración y puesta en marcha de recursos docentes (herramientas y contenidos). (b) Acompañar en la implementación del nuevo modelo educativo, el plan estratégico, los proyectos de innovación docente y el plan director de servicios (evolución del aula). (c) Asegurar la estabilidad, evolución y funcionamiento de las aulas y recursos docentes (editor de plan docente, diseño del aula, materiales docentes). (d) Conocer las necesidades y características de 
estudiantes y profesorado para mejorar las experiencias de aprendizaje y de enseñanza.

2. Un equipo de desarrollo y mantenimiento que gestione la calidad del desarrollo y el servicio de mantenimiento de las aplicaciones. Sus funciones son: (a) Atender los servicios generales del campus (página de inicio, foros, buzón, red de personas). (b) Velar por la accesibilidad y los temas de móviles. (c) Participar en el proyecto sobre la experiencia global de usuario del estudiante. (d) Consolidar el campus virtual. (e) Introducir herramientas de red social en el campus. (f) Trabajar en la difusión internacional de los proyectos, para buscar nuevas fuentes de financiación.

3. Un equipo de integración que realiza el diseño tecnológico y la verificación de la integración y la calidad de las aplicaciones. Sus funciones son: (a) Coordinación con la Biblioteca. (b) Definir y ofrecer un conjunto de servicios a las aplicaciones de Campus Virtual basados en estándares, software libre y que ofrezcan la interoperabilidad. (c) Evolucionar y mejorar las herramientas base del CV hacia nuevos servicios (autentificación, administrador y servidor de correo). (d) Ejecutar la primera fase del proyecto de I + D con financiación externa: Learning Apps. (e) Revisar el apoyo a los clientes del CV y buscar acciones de mejora para reducir el número de incidencias.

4. Un equipo de ayuda al usuario que gestiona la tecnología para el trabajo y proporciona indicadores de calidad. Sus funciones son: (a) Concluir los proyectos y analizar los resultados obtenidos. (b) Apoyar las iniciativas de mejora y compatibilización. (c) Consolidar el grupo y las nuevas responsabilidades. (d) Detectar puntos críticos. (e) Promover la innovación tecnológica y la diversidad. (f) Detectar oportunidades de mejora en otras áreas de la IED.

\section{Política profesoral}

En el marco de las necesidades para el mejoramiento de la calidad académica institucional en la IED se enfatiza en la formación de profesores en las dimensiones: disciplinar y científica, riqueza en valores y cultural, formación pedagógica y en nuevas tecnologías de la información y comunicación y con ello dar cuenta del fortalecimiento del modelo curricular, de la alta cualificación del talento humano, de la efectividad 
de las acciones formativas en lo que respecta a la satisfacción, los conocimientos adquiridos, la transferencia de conocimientos, habilidades y actitudes luego de la capacitación recibida y de los resultados finales que ocurren en los docentes referidos a la mejora de la calidad en el diseño de cursos virtuales (Vela, 2013).

Propósito. El propósito de integrar al cuerpo de docentes es garantizar la organización y oferta de los diferentes niveles educativos ofrecido por la IED en sus diferentes áreas. Su desempeño consistirá en la elaboración y gestión de propuestas del mejoramiento de los programas académicos.

Definición. Los docentes de la IED están representados por un conjunto de profesores investigadores que se integran a partir proyectos de investigación. Aportan propuestas teórico-metodológicas para generar y aplicar conocimientos especializados.

Ingreso y permanencia. Se consideran las siguientes políticas:

1. La IED garantiza el ingreso de una planta de profesores con formación en maestría, doctorado y manejo de una segunda lengua para fortalecer una visión intercultural e internacional. 2. La IED propende por una planta de profesores estable. El $70 \%$ tendrá una dedicación de tiempo completo, $20 \%$ de medio tiempo y un $10 \%$ por horas acorde a las necesidades del servicio docente contribuyendo al aseguramiento de los sistemas de docencia, investigación y proyección social.

3. La formación, vigencia y mantenimiento de los conocimientos de los profesores está sujeta a las exigencias y prioridades profesionales y disciplinares de los currículos, la investigación y la proyección social.

4. La IED está comprometida con la formación pedagógica de sus profesores para fortalecer la búsqueda de nuevas alternativas de enseñanza aprendizaje acordes con el desarrollo del conocimiento y las nuevas tecnologías.

5. La IED propende por una formación profesoral fundamentada en valores y el respeto por las diferencias culturales.

Desarrollo de la docencia. Se consideran:

1. Promover estrategias pedagógicas innovadoras que medien los procesos de enseñanza aprendizaje significativo, 
creativo y colaborativo.

2. Propiciar un modelo de evaluación por competencias coherenteconlosprincipios deinvestigación, contextualización, interdisciplinariedad, flexibilización y participación.

3. Fomentar espacios de praxis pedagógica permanente que posibiliten el mejoramiento del que hacer propio del docente.

4. Garantizar la formación integral de los estudiantes y egresados como agentes de transformación social, cultural y ambiental.

5. Propender permanentemente por la formación de un aprendizaje autónomo que garantice la capacidad crítica y toma de decisiones frente a situaciones del contexto académico y social.

6. Impulsar la formación integral a partir de procesos de investigación, innovación y emprendimiento que respondan a los intereses y a problemáticas del contexto.

7. Incentivar la innovación en todos los procesos académicos buscando generar una cultura institucional.

8. Promover estrategias que permitan potencializar las competencias genéricas y específicas como escenarios de prácticas laborales.

Internacionalización. Se consideran las siguientes políticas:

1. La IED está comprometida con la gestión de convenios con instituciones de educación superior nacionales e internacionales con el objetivo que se generen comunidades científicas y movilidad con fines académicos.

2. Apoyar la participación de los profesores en la participación de ponencias en congresos Nacionales e Internacionales.

Evaluación del desempeño docente. La IED busca contribuir al mejoramiento del desempeño laboral de los profesores. Se constituye en una herramienta clave en la gestión del talento humano y sus resultados se utilizaran para la toma de decisiones en cuanto a promoción interna de personal, capacitaciones, planes de desarrollo y desvinculaciones.

La evaluación docente permitirá el mejoramiento de los profesores en sus competencias de enseñanza, en la promoción del aprendizaje autónomo en sus estudiantes y en su desarrollo personal y profesional 
al informarle sobre los aspectos que debe mejorar para que cada vez sea más eficaz en su aporte al desarrollo institucional en la docencia, la investigación y la proyección social.

Para el Ministerio de Educación Nacional (MEN) de Colombia, la evaluación del desempeño laboral de docentes y directivos docentes hace parte de un compromiso nacional con la calidad de la educación, que debe propiciar la reflexión permanente, tanto individual como colectiva, sobre las posibilidades de crecimiento personal y profesional del talento humano encargado de dirigir las instituciones educativas y de liderar los procesos de enseñanza y aprendizaje en las aulas colombianas (MEN, 2011). En tal perspectiva,

Según Valdez (2000) la evaluación del desempeño docente se concibe como una actividad de análisis, compromiso y formación del profesorado, que valora y enjuicia la concepción, práctica, proyección y desarrollo de la actividad y de la profesionalización docente.

Actualmente, las instituciones universitarias deben procurar establecer políticas y formas eficientes para elevar el nivel de sus docentes y establecer evaluaciones para evidenciar el desempeño del personal académico en correspondencia con ese nivel deseado de excelencia y calidad al considerar a toda evaluación como un proceso para estimular - juzgar el valor, la excelencia, las cualidades de alguna persona (Colina, Z., Medina, N., Parra, D., Cendrós, J. \& Montoya, C., 2008; Chiavenato, 2000).

El profesor prevalece como uno de los indicados ya que influye decisivamente en el producto y por ello ha sido objeto de evaluación (Cantón, 2001). Jiménez (2008) considera que la complejidad del concepto de desempeño docente se debe a que es, igual que muchos otros fenómenos psicológicos y sociales, una acción humana imposible de aislar para estudiarla y evaluarla en un contexto particular. Por lo tanto, el desempeño docente, por ser una actuación del hombre, es observable y mensurable y lo primordial es saber que cuando se habla de medir es, como dice Chiavenato (2000), el comportamiento de rol del docente.

Gago (2002) plantea que sólo evaluando es posible identificar la medida en que se logran los propósitos, se conocen las causas del buen éxito o del fracaso, permite tomar decisiones para mejorar; además, es un hecho inevitable, pues de una u otra forma siempre se evalúa. En la 
medida en que "la necesidad por evaluar se encuentre mejor delimitada, los objetivos de estudio serán entonces más claros, las preguntas por responder más precisas, y los métodos y técnicas por aplicar mejor seleccionados" (Valenzuela, 2005, p.49). Para Montenegro (2003, p.31), "tener un modelo es contar con una estructura que permita evaluar el desempeño con parámetros previamente establecidos, de tal manera que el docente sepa de antemano qué se le va a evaluar, cómo, cuándo y para qué".

Estímulos al desempeño de gestión académica. Los planes de estímulos al desempeño de gestión académica de la IED se orientan a dar reconocimientos por el desempeño y aporte en las áreas de investigación, docencia y proyección social.

Bienestar y calidad de vida. Se consideran las siguientes políticas:

1. La IED propende por una política de salud y seguridad para su personal orientada a maximizar la salud y bienestar tanto físico como mental.

2. La IED promoverá estilos de vida saludables que contribuyan a mejorar la calidad de vida y la de sus familias mediante el desarrolla de actividades sociales, deportivas y recreativas.

\section{Políticas para los estudiantes}

Para Simonson (como se citó en Moore, 2007), las políticas de los estudiantes deben integrarse y verse reflejadas en los servicios ofrecidos relativos a la modalidad de Educación a Distancia. Sin embargo las políticas regulares pueden necesitar ser modificadas para acomodar el estudiante a distancia, específicamente cuando se necesita de una instrucción asincrónica.

Para brindar este soporte del estudiante se hace necesario proveer a la IED de los siguientes servicios: admisiones, asesoramiento, tutoría virtual, financiamiento, cuentas de acceso al campus virtual, servicios de biblioteca virtual, entrega de materiales, tramitación de reclamaciones de los estudiantes, entre otros (Simonson, Smaldino, Albright y Zvacek, 2009). 
En esta área se han determinado los siguientes procesos que favorecen el aprendizaje del estudiante y corresponden a:

Definición de los perfiles de ingreso/egreso, admisión y matriculación de los estudiantes. En el proceso de diseño del programa de formación, se establecerán los perfiles de ingreso y egreso acordes con la política institucional. Corresponde al área de incorporación y seguimiento del estudiante la responsabilidad de los procesos de matrícula de los estudiantes, mientras que la admisión cuenta además con la responsabilidad de la secretaría en la validación de dicho trámite académico.

Proceso de acceso y matrícula. El proceso de acceso y matrícula es responsable de facilitar el ingreso a la IED de los nuevos estudiantes. EI área incorporación y seguimiento del estudiante, una vez finalizado el proceso para el ingreso de nuevos estudiantes, debe evaluar los resultados del proceso: número de solicitudes, número de admitidos, número de matriculados, media de créditos matriculados, en global y para cada uno de los programas, además de analizar los resultados de satisfacción de los estudiantes con el proceso de incorporación. A partir de la evaluación de estos resultados se deben establecer acciones de mejora.

Asignación de perfiles de acceso al campus virtual. Todo estudiante matriculado en la IED debe tener asignado un usuario y contraseña para poder acceder al campus virtual de la universidad y de la misma manera a los servicios ofrecidos en su proceso de formación como cursos virtuales, servicio de biblioteca virtual, bienestar universitario, procesos de admisión y matricula, preguntas, quejas, reclamaciones y sugerencias, entre otros.

Apoyo y orientación al estudiante, metodología de enseñanza y evaluación de los aprendizajes. La IED debe ser consciente, desde el inicio de sus actividades, de la conveniencia de tener un sistema de orientación capaz de dar respuesta a las necesidades específicas de los estudiantes en un entorno de formación virtual. Este modelo debe ir evolucionando en función de las nuevas necesidades del sistema universitario y de las innovaciones tecnológicas.

Desde el primer momento en que el estudiante solicita el acceso a la IED debe recibir el apoyo del servicio al estudiante, el que cuenta con un docente, que le guía en todos los procesos de matriculación de los 
cursos y debe disponer de un servicio de información (navegación) por el Campus Virtual de la IED. También debe recibir el apoyo del docente colaborador que lo acompaña en su proceso de aprendizaje.

Plan de tutoría de programa. El modelo IED debe incluir unos mecanismos de apoyo y orientación al estudiante. Estos mecanismos se concretan en las acciones que se llevan a cabo desde el equipo de servicio al estudiante y desde el desarrollo del plan de tutoría. Los diferentes programas se deben dotar de un plan de tutoría de incorporación, de inicio y uno de seguimiento; en ellos se determinan las características de la acción tutorial en las diferentes fases de la trayectoria académica del estudiante y también en los diferentes momentos de la actividad del semestre. Este plan de tutoría se debe ajustar a la singularidad de cada uno de los cursos y se debe elaborar a partir de las directrices que da el director del programa. La propuesta del plan de tutoría del programa debe ser preparada por un docente con el apoyo del equipo de función tutorial; el director de programa es el que aprueba el plan de tutoría semestral. Así mismo, son los docentes los que tienen la función de llevar a cabo el plan de tutoría a lo largo del semestre, a través de las aulas de tutoría del campus virtual.

Prácticas externas y movilidad de los estudiantes. Las prácticas externas son el escenario adecuado para poner en práctica, especialmente, los conocimientos asociados a las competencias de tipo procedimental y actitudinal (intrapersonales e interpersonales), y para reforzar la adquisición de estas mediante el aprendizaje experiencial. El desarrollo y seguimiento de las prácticas debe contar con el apoyo de un docente colaborador y de otros docentes.

Movilidad de los estudiantes. El plan de movilidad de la IED se irá desarrollando a medida que el sistema universitario se adapte a la nueva estructura de las enseñanzas y a la nueva metodología de enseñanzaaprendizaje por la que las competencias constituyen un elemento esencial en el aprendizaje. Dichas propuestas de movilidad para cada grado se deberán presentar anualmente desde la comisión académica; una vez consensuados para cada programa, estos programas de movilidad deberán ser presentados al Consejo Directivo para su aprobación.

Orientación profesional de los estudiantes. El perfil del estudiante de la IED corresponde a una persona mayoritariamente adulta. La motivación para estudiar en la IED responde a una necesidad de mejora profesional, 
o a la voluntad de ampliar unos conocimientos y poder llevar a cabo una mejor práctica profesional, o para complementar su formación con conocimientos de otras disciplinas. Por esta razón, los servicios de orientación profesional no deben ir tan orientados a la búsqueda del primer empleo, sino a facilitar la interrelación entre los miembros de la IED y las empresas, y así crear una red de contactos que faciliten el intercambio y la mejora profesional. También se deben llevar a cabo acciones específicas en función de las necesidades emergentes en el mercado laboral, las necesidades expresadas por los propios estudiantes o las sugerencias que aportan los diferentes estudios de la IED. Dichas acciones tienen por objetivo dar a conocer nuevos enfoques o necesidades profesionales.

Sistema de alegaciones. La responsabilidad de los criterios de resolución de las alegaciones recae en los directivos, pero la información y la comunicación al estudiante se realizan a través del grupo de atención al estudiante. El área de servicio al estudiante debe elaborar al final de cada semestre un informe de las alegaciones presentadas. La evaluación del proceso y las acciones de mejora son cometido tanto de los directivos como del área responsable.

Sistema de reclamaciones, quejas y sugerencias. Dicho sistema, debe seguir las mismas acciones que el servicio de atención a los estudiantes en las que se considera el espacio virtual de comunicación con ellos, y lo hace en relación con todos los trámites y las actividades vinculadas con su actividad académica.

\section{Conclusiones}

La creación de instituciones y de programas que se oferten en la modalidad de educación a distancia apoyada en tecnología requiere de diseños sistémicos de los servicios que pretende ofertar en el sector educativo dentro de un país tiene que estar en articulación a las consideraciones que el estado y la sociedad disponen de lo educativo. Por lo general, se tiene un sistema educativo regulado al que debe también vincularse por ser lo educativo un servicio público con las implicaciones que eso implica.

La visión sistémica de la institución vista desde las seis áreas discutidas en el artículo, permite la articulación de lo filosófico, como elemento 
diferenciador del servicio educativo por ofertar, las características que se propone formar en los educandos, en los rasgos diferenciales de su comunidad educativa y en la identidad propia de la institución educativa, con el quehacer, las relaciones con el contexto social local, regional, nacional e internacional donde se requieran sus servicios educativos y en el impacto que tendrá sobre esas comunidades.

La construcción de política para cada subsistema, si bien tiene unos referentes generales como se presentaron en este artículo, deben ser adaptados a las condiciones institucionales, de comunidad académica y disponibilidad de recursos que cada proyecto institucional pretenda ofertar; sin embargo, el principio rector es la articulación estricta con la filosofía institucional garantizando su coherencia e identidad de egresados, estudiantes, docentes y administrativos que actúan en el desarrollo de la sociedad de la que hacen parte.

De los miembros de la comunidad educativa, especial énfasis tendrán los estudiantes como el centro de la actividad de la comunidad universitaria a distancia, de la disponibilidad permanente de los servicios académicos y administrativos comprometidos por la IED. Esto porque son el talento humano en formación que luego en su carácter de egresados va a contribuir al desarrollo social de su comunidad, de su país, con las notas distintivas que el proyecto formativo de la IED le inculcaron.

En la misma forma, los docentes es el otro estamento de vital importancia para la IED, no sólo por desarrollar el proyecto formativo de los programas en oferta, sino también porque ayudan al reconocimiento institucional mediante aportes en investigación y desarrollo, la extensión universitaria y la proyección internacional entre otros aspectos de la vida académica de la universidad.

Como grupo social, la IED debe vivir dentro de una cultura de calidad, de observancia de los fundamentos organizacionales inspirados en la filosofía institucional, en la transparencia y en la eficiencia y la eficacia como manifestaciones plenas de una cultura de calidad. 


\section{Referencias bibliográficas}

Acevedo Zapata, S. (2014). Inclusión digital y educación inclusiva. Aportes para el diseño de proyectos pedagógicos con el uso de tecnologías de la comunicación. Revista De Investigaciones UNAD, 13(1), 41-57. doi:http://dx.doi.org/10.22490/25391887.1130

Acevedo Zapata, S. (2015). La formación de profesionales desde la reflexión en la acción con elementos fundamentales de la pedagogía crítica. Revista De Investigaciones UNAD, 14(1), 151 - 169. doi:http://dx.doi.org/10.22490/25391887.1350

Álvarez, S. (1992). Evaluación de calidad como alternativa para la transformación de las universidades: el caso de Argentina. Revista Interamericana de Gestión Universitaria (3).

Amaya Balaguera, Y. (2015). Guía metodológica ágil, para el desarrollo de aplicaciones móviles "AEGIS-MD". Revista De Investigaciones UNAD, 14(1), 97 - 113.

Arévalo, M., \& Gamboa, A. (2015). TIC en el currículo de matemáticas. Una orientación desde el marco de las políticas y proyectos educativos. Revista Interamericana de Investigación, Educación y Pedagogía, RIIEP, 8(1). doi:http://dx.doi.org/10.15332/s1657107X.2015.0001.07

Ausubel, D. (2002). Adquisición y retención del conocimiento: una perspectiva cognitiva. Madrid: Paídos.

Bonilla, M. (2004). Educación virtual: Nuevo paradigma en el proceso de enseñar y aprender. Hato Rey, PR: Publicaciones Puertorriqueñas, Editores.

Bonvecchio, A. M., \& Maggioni, B. E. (2004). Evaluación de los aprendizajes. Buenos Aires: Novedades Educativas y Universidad Nacional del Cuyo.

Bornas, J. (1994) La autonomía personal en la infancia. Estrategias cognitivas y pautas para su desarrollo. España, Siglo XXI editores.

Bruns, B. \& Luque, J. (2015), Great Teachers: How to Raise Student Learning in Latin America and the Caribbean. Washington, DC, Banco Mundial. doi:10.1596/978-1-46480151-8.

Chiavenato, I. (2000). Administración de recursos humanos. Bogotá: Mc Graw-Hill, Colina, Z., Medina, N., Parra, D., Cendrós, J. \& Montoya, C. (2008). Modelo para la evaluación del desempeño docente en la función docencia universitaria. Revista Investigación Educativa.12(22), 99-126. ISSN 17285852

Copertari, S., Sgrecia, N., \& Segura, M. L. (2011). Políticas universitarias, gestión y formación docente en Educación a Distancia. Hacia una pedagogía de la virtualización, RED. Revista de Educación a Distancia, (27), 1-16. Recuperado de: http://www.redalyc. org/src/inicio/ArtPdfRed.jsp?iCve=54719121003

Dick, W. Carey, L. \& Carey, J. (2009). The systematic design of instruction. (7a. Ed.). N. J.: Upper Saddle River. Pearson. 
Ferreyra, H., Castagno, F., Salgueiro, M., \& Tucci, S. (2012). Significados y efectos en torno a la evaluación de los aprendizajes escolares. Revista Interamericana De Investigación, Educación y Pedagogía, RIIEP, 5(2). doi:http://dx.doi.org/10.15332/s1657107X.2012.0002.05

Fliguer, J.L. (2010). Fundamentos de las políticas públicas en educación superior. Problematización de las políticas de expansión en Argentina. Revista Científica de UCES, XIV (2), 49-63. Recuperado de: http://dspace.uces.edu.ar:8180/dspace/ bitstream/123456789/894/1/Fund_de_las_politicas_Fliguer.pdf

Flórez, R. (1994). Hacia una pedagogía del conocimiento. Santafé de Bogotá: Editorial McGraw- Hill

Gago, A. (2002). Apuntes acerca de la Evaluación Educativa. México DF: Secretaría de Educación Pública,.

Gamboa, M., Sandoval, Y. \& Ahumada, V. (2017). Diseño de Ambientes de EnseñanzaAprendizaje: Consideraciones con base en la PNL y los Estilos de Aprendizaje. Bogotá D.C.: Ediciones Universidad Simón Bolívar y Sello Editorial UNAD. Recuperado de http:// hdl.handle.net/10596/11973

Garrison, D., \& Anderson, T. (2005). El e-learning en el siglo XXI: investigación y práctica. Barcelona: Ediciones Octaedro.

Gellman-Danley, B. \& Fetzner, M. (1998). Asking the really tough questions: Policy issues for distance learning. Recuperado de http://www.westga.edu/ distance/danley11.html. Institute

Gimeno-Sacristán J. \& Pérez-Gómez, Á. (1992). Comprender y transformar la enseñanza. Madrid: Morata.

Jiménez, J. A. (2008). Cuatro modelos de evaluación docente. PsicoPediaHoy, 10(9). Disponible en: http://psicopediahoy.com/evaluacion-docente-cuatro-modelos/.

Jiménez Mendoza, W., Luciano Alipio, R., \& Soto Carrión, C. (2014). Influencia de las fuentes de financiamiento externo en el crecimiento y desarrollo de las empresas agroindustriales del distrito de Abancay Apurímac Perú (periodo 2012- 2013). Revista De Investigaciones UNAD, 13(1), 261-273. doi:http://dx.doi.org/10.22490/25391887.1141

King, J., Nugent, G., Eich, J., Mlinek, D., \& Russell, E. (2000). A policy framework for distance education: A case study and model. DEOS NEWS 10(10). Recuperado de www. schoolofed.nova.edu/dll/Module3/Policy-Framework.doc.

Klimenko, O. \& Alvares, J. (2009). Aprender cómo aprendo: la enseñanza de estrategias metacognitivas. Educación y Educadores, 12(2), 11-28. Recuperado de: http://www. redalyc.org/pdf/834/83412219002.pdf

Lamas, R. H. (2008). Aprendizaje autorregulado, motivación y rendimiento académico. LIBERABIT, (14), 15-20. Recuperado de http://www.scielo.org.pe/pdf/liber/v14n14/ a03v14n14.pdf. 
Lampert, E. (2000). Educación a Distancia ¿elitización o alternativa para democratizar la enseñanza? Perfiles educativos, (88), Recuperado de: http://redalyc.uaemex.mx/redalyc/ pdf/132/13208805.pdf

Matias, P. J. (2008). Políticas públicas de educação no Brasil: A utilização da ead como instrumento de inclusão social. Journal of Technology Management \& Innovation, 3(02), 44-55. Recuperado de: http://redalyc.uaemex.mx/pdf/847/84730205.pdf

Miklos, T., \& Arroyo, M. (2008). Una visión prospectiva de la Educación a Distancia en América Latina. Innovación Educativa, 8(42), 5-17. Recuperado de: http://www.redalyc. org/src/inicio/ArtPdfRed.jsp?iCve=179421234002

Ministerio de Educación Nacional (MEN) (2011). Evaluación anual de desempeño de docentes y directivos docentes. Recuperado de http://www.mineducacion.gov.co/1759/ w3-article-246098.html

Montenegro, I. (2003). Evaluación del Desempeño Docente. Fundamentos, modelos e instrumentos. Bogotá: Magisterio.

Moore, M.G. (2007). (Ed). Handbook of Distance Education. Mahwah, New Jersey: Lawrence Erlbaum Associates, Inc.

Nagles, J \& Calderón, J. (2016) Currículo: en búsqueda de precisiones conceptuales. Revista Educación \& Pensamiento. 23(23). Recuperado de https://dialnet.unirioja.es/ descarga/articulo/5740421.pdf

Nitko, A. J. \& Brookhart, S. M. (2011). Educational assessment of students. (6a. Ed.). Boston. M. A.: Pearson.

Ormrod, J. E. (2012). Human Learning. (6a. Ed.). Allyn \& Bacon: Upper Saddle River, NJ.

Ortiz, J. 2010). Comunidades de aprendizaje. En resúmenes de políticas educativas, (proyecto USAID/Reforma Educativa en el Aula. Guatemala

Pallof, R. \& Pratt, K., (2007). Lessons learned and look ahead. En: Building online learning communities. (2th Ed.). (pp. 227-238). San Francisco, CA: Jossey Bass.

Panitz, T. (2001) Collaborative versus cooperative learning a comparison of the two concepts which will help us understand the underlying nature of interactive learning. Recuperado de: http://home.capecod.net/ tpanitz/tedsarticles/coopdefinition.htm

Piskurich, G. (2006). E-learning fast, cheap and good. Performance Improvement, 45(1), 18-23. Recuperado de la base de datos ProQuest.

Régio, L., Egry, E., \& Apostólico, M. (2015). Consideraciones acerca de la importancia del estudio de saberes necesarios para el afrontamiento de la violencia infantil en la atención primaria de salud.. Revista De Investigaciones UNAD, 14(2), 137-149. doi:http://dx.doi. org/10.22490/25391887.1463 
Restauri, S. (2004). Creating an effective online distance education program using targeted support factors. TechTrends, 48(6), 32-39. Recuperado de la base de datos Wilson Web.

Reynaga, S, \& Farfán, P. (2004) Redes académicas, potencialidades académicas. Cuarto Congreso Nacional y Tercero Internacional: "Retos y Expectativas de la Universidad". Guadalajara: México. Recuperado de http://www.anuies.mx/media/ docs/89_2_1_1012161230Reynaga_Obregon_y_Farfan_Flores_Redes_Academicas.pdf

Rodríguez, L \& Zevallos, C. (2003). Antología sobre evaluación institucional. Lima, Perú: Universidad Nacional de Educación (UNE). Recuperado de http://www.une.edu.pe/ acreditacion/images/evaluacion\%20institucional_modificado.pdf

Rojas Mesa, J., \& Leal Urueña, L. (2014). Entre flujos y fronteras: la educación superior mediada tecnológicamente vista a través de una perspectiva etnográfica. Revista De Investigaciones UNAD, 13(2), 9-27. doi:http://dx.doi.org/10.22490/25391887.1143

Rubio, G. G., \& González, R. J. (2011). Leyes, normas y reglamentos que regulan la educación superior a distancia y en línea en Colombia. En: Barbosa, C. J. (Ed.). Leyes, normas y reglamentos que regulan la educación superior a distancia y en línea en América Latina y el Caribe. Loja, Ecuador.: Universidad Técnica Particular de Loja. Recuperado de: http://www.utpl.edu.ec/caled/images/leyesnormas.pdf

Sandia, B. \& Jonás, Montilva, J. (s.f.). Aspectos Metodológicos para el Desarrollo de Programas de Estudios Interactivos a Distancia Basados en WEB. Recuperado de 2011 de http://www.saber.ula.ve/bitstream/123456789/15844/1/04_aspectos_metodo.pdf

Simoneau, R. (2003). La evaluación institucional. Universidad de Quebec. Antología sobre evaluación institucional. p. 50-56 Recuperado de http://www.une.edu.pe/acreditacion/ images/evaluacion\%20institucional_modificado.pdf

Simonson, M. (2007). Institutional policy issues. En: Moore, G. M. (Ed.) Handbook of distance education. (2a. Ed.). Mahwah, NJ: Lawrence Erlbaum Associates, Publishers.

Simonson, M., Smaldino, S., Albright, M. \& Zvacek. S. (2012). Teaching and learning at a distance: Foundations of distance education. (5th Ed.). Boston, MA: Allyn \& Bacon.

Valdés, V. H. (2000). En un mundo de cambios rápidos, sólo el fomento de la innovación en las escuelas permitirá al sistema educacional mantenerse al día con los otros sectores. Ponencia presentada en el Encuentro Iberoamericano sobre Evaluación del Desempeño Docente. OEI, México, 23 al 25 de mayo. Disponible en: http://www.oei.es/de/rifad01. htm

Valenzuela, J. (2005). Evaluación de instituciones educativas. México: Editorial Trillas,. Valenzuela, J. (2010). La evaluación de la calidad en la educación a distancia. Revista Didasc@lia: Didáctica y Educación. ISSN 2224-2643. Recuperado de https://dialnet. unirioja.es/descarga/articulo/4227280.pdf 
Vela, P. (2013) Evaluación de acciones formativas en el diseño de cursos en ambiente e-learning en una universidad a distancia colombiana [Tesis Doctoral].

Vela, P., Ahumada, V., \& Guerrero, J. (2016). Conceptos estructurantes de la Educación a Distancia. Revista De Investigaciones UNAD, 14(1), 115 - 149. doi:http://dx.doi. org/10.22490/25391887.1349

Watson, J. \& Gemin, B. (2009). Promising practices in online learning: Policy and funding frameworks for online learning. International Association for K-12 Online Learning. Recuperado de http://www.inacol.org/research/promisingpractices/.

Yang, J. (2006). The discussion of media selection and accessible equity in distance education. The Journal of American Academy of Business, 10(1), 126-130. Recuperado de la base de datos ProQuest.

\section{Apéndice. Normatividad Colombiana}

\section{Ley 115 de 1994 General de Educación}

Esta ley Indica las normas generales que organizan el servicio público de la educación en Colombia. Le indica a los entes encargados cómo debe llevarse a cabo la prestación de la educación. La Educación Superior se regula por una ley especial que no puede ir en contra de lo dispuesto en forma general en la ley 115. Se puede acceder a esta en http://www. col.ops-oms.org/juventudes/Situacion/LEGISLACION/EDUCACION/ EL11594.HTM

\section{Ley 30 de 1992}

En este se organiza el servicio público de la educación superior. Se desarrolla el principio de autonomía universitaria acorde a la constitución política del país. Se establecen los objetivos y campos de acción de los programas de pregrado y posgrado. Se organiza la educación superior en tres grupos: instituciones técnicas profesionales, instituciones universitarias o escuelas tecnológicas y universidades. La Ley establece también los criterios y competencias del Estado en materia de fomento, inspección y vigilancia del sistema de educación superior. Se puede acceder a esta información en el link: http://www.dmsjuridica.com/ CODIGOS/LEGISLACION/LEYES/Lo030_92.htm 


\section{Decreto 1075 de 2015}

Con este se reglamenta el registro calificado para la oferta y programas académicos de educación superior. Es el instrumento del sistema de aseguramiento de la calidad de la educación superior con la que el estado verifica el cumplimiento de las condiciones de calidad. Se puede acceder a este en el siguiente link: http://www.mineducacion.gov.co/ normatividad/1753/w3-article-351080.html

\section{Decreto 1279 de junio 19 de 2002}

En el que se establecen las condiciones salariales y prestacionales de los profesores de las Universidades estatales. Se puede acceder a esta información en el link: http://www.unal.edu.co/dnp/Archivos_base/ decreto1279.pdf

\section{Propiedad intelectual}

En Colombia la propiedad intelectual es la denominación que recibe la protección legal sobre toda creación del talento o del ingenio humano en los campos científico, literario, artístico, Industrial o comercial.

La protección intelectual es de tipo jurídica y en Colombia las leyes existentes no tienen esta denominación. Hay dos denominaciones diferenciadas: Derecho de autor que puede visitarse en http://www.derechodeautor.gov.co/htm/legal/legislacion/leyes_arch/23. pdf y propiedad industrial a la que puede accederse en http://www.sic. gov.co/recursos_user/documentos/compendio/Propiedad_Normas.pdf

\section{Normativa del gobierno electrónico en Colombia}

Se presenta en esta el programa de gobierno en línea del Ministerio de Tecnologías de la Información y las Comunicaciones que contiene el marco general normativo, normativa sobre acceso a la información, normativa sobre participación ciudadana, normativa sobre privacidad, habeas data y protección de datos, normativa sobre seguridad de la información, normativa sobre interoperabilidad, normativa sobre registros, expedientes y notificación electrónica, normativa sobre documento y firma digital / electrónica y la normativa sobre contratación electrónica. Se puede acceder a esta información en el link: http://programa.gobiernoenlinea.gov.co/apc-aa-files/5686d2a87532a21 a7oead773ed71353b/NormativaGEL.pdf 